\title{
Prevención Primaria como aprendizaje experiencial real.
}

\author{
Carla de Paredes $^{\mathrm{a}}$, Lucia Aparicio ${ }^{\mathrm{b}}$, Elisa Gimenez ${ }^{\mathrm{c}}$ y Cristina Escamilla ${ }^{\mathrm{d}}$
}

anuniversidad Europea de Valencia carla.deparedes@universidadeuropea.es, ${ }^{b}$ Universidad Europea de Valencia, lucia.aparicio@universidadeuropea.es y ${ }^{\mathrm{c}}$ Universidad Europea de Valencia elisa.gimenez@universidadeuropea.es, ${ }^{\mathrm{d}}$ Universidad Europea de Valencia, mariacristina.escamilla@universidadeuropea.es

\section{Abstract}

This paper presents an opportunity for university students for "experiential learning", with the aim of developing a project aimed at vulnerable children with the aim of a primary prevention tool in school. A transversal theme was selected in subjects of the Double Degree of Criminology and Psychology, the "Child Grooming", focused on a real situation, beyond the "simulation". It is intended that children learn strategies to identify and avoid certain dangers in the network.

The methodology consisted in the development by consecutive phases: 1st Unification Criteria with the institution where the activity was carried out; 2nd Development and elaboration of the tool; 3rd. Simulation; 4th Real situation. The results of the activity were very satisfactory, from learning to usefulness.

The conclusions, the implementation of this methodology was very satisfactory for several reasons: to carry out the practice of what was learned and work in relation to the degree; for practicing the future profession in one of its areas such as "prevention"; for the feeling of being using friends who contribute to the community, and as a motivation to continue studying, learning and growing in the professional field.

Keywords: prevention, learnig by doing, real experiential learning, social sciences

\section{Resumen}

En este trabajo se presenta una actividad a los estudiantes universitarios mediante el "aprendizaje experiencial”, con el que desarrollar un proyecto dirigido a menores vulnerables con el fin de establecer una herramienta de prevención primaria en la escuela. Se seleccionó un tema transversal en asignaturas del Doble Grado de Criminología y Psicología, el "Child Grooming”, enfocado hacia una situación real, más allá de la "simulación". Se pretende que los menores aprendan estrategias de identificación y evitación de ciertos peligros en la red.

La metodología consistió en desarrollo por fases consecutivas: $1^{a}$ Unificación criterios con la institución donde se llevó a cabo la actividad; $2^{a}$ Desarrollo y elaboración de la herramienta; $3^{a}$. Simulación; $4^{a}$ Situación real. Los resultados de la actividad fueron muy satisfactorios, desde el aprendizaje, hasta la utilidad.

Las conclusiones, la puesta en marcha de esta metodología fue muy satisfactoria por varias razones: por llevar a cabo la práctica de lo aprendido y elaborado en relación con la titulación; por practicar la futura profesión en uno de sus ámbitos como es la 
"prevención"; por la sensación de sentirse útiles aportando su contribución a la comunidad, y como motivación para seguir estudiando, aprendiendo y creciendo en el campo profesional.

Palabras clave: child grooming, prevención, learnig by doing, aprendizaje experencial, ciencias sociales

\section{Introducción}

Hoy en día estamos asistiendo a nuevas formas de enfocar la educación de nuestros estudiantes, gracias en parte a la implantación del Espacio Europeo de Educación Superior (EEES) que promueve la mejora del sistema educativo universitario, al mismo tiempo que fomenta una mayor implicación del estudiante en su propio proceso de aprendizaje. En este sentido, los sistemas educativos van evolucionando, fomentando continuas transformaciones y las nuevas metodologías de aprendizaje se adaptan para poder dar respuesta a las nuevas necesidades y demandas (Benito y Cruz, 2005).

La docencia tradicional, basada en clases magistrales presenciales, ha ido perdiendo cada vez más influencia por lo que se apuesta por procesos de aprendizaje basados en otro tipo de metodologías docentes, dirigidas a valorar un conjunto de oportunidades y condiciones que ofrecer a los estudiantes y así promover su aprendizaje (De Miguel, 2005; Labrador y Andreu, 2008). Esta nueva conceptualización implica necesariamente un cambio en el rol del profesor y del alumno, fomentando la participación activa del alumno y restando protagonismo a la labor del docente (Miguel-Dávila, López-Berzosa y Martín-Sánchez, 2012).

Dentro de estas innovaciones docentes, el aprender a hacer se acomoda dentro de este cambio de perspectiva que transita la concepción de la docencia basada en la enseñanza a otra centrada en el aprendizaje (Zabalza, 2011). Esto permite que la formación del alumno no se base únicamente en la adquisición de conocimientos, que pueden ser de carácter teórico o práctico, sino también promueven el desarrollo de competencias y habilidades que les proporcionan las herramientas necesarias para poder prosperar en una sociedad cada vez más compleja (Aparicio y De Paredes, 2016).

Para implantarlo plenamente y con éxito es necesaria la utilización de herramientas que promuevan el aprendizaje constructivo y creativo de los alumnos. De este modo, los alumnos podrán desempeñar un papel más activo, en lugar de ser meros receptores de conocimientos, convirtiéndose así en los auténticos protagonistas de su proceso formativo.

Un gran aporte al proceso de enseñanza-aprendizaje es el "aprendizaje experiencial” que se presenta como una metodología educativa con un especial potencial para responder a los nuevos retos a los que se enfrenta la educación superior universitaria en estos momentos, en especial en relación con las nuevas demandas competenciales y conceptuales y la obtención de una formación lo más realista posible a los nuevos modelos del mundo laboral (Ariza, 2010; March, 2006).

(c) ) EY-NC-ND 2018, Universitat Politècnica de València

Congreso IN-RED (2018) 
En este sentido, el aprendizaje experiencial, basado en el pionero modelo de Dewey (1938) y retomado posteriormente por diferentes autores (Chisholm, Harris, Northwood y Johrendt, 2009; Itin, 1999) enfatiza elementos distintivos como la experimentación fuera del aula que permite una verdadera interacción con el medio y una aplicabilidad del conocimiento al contexto de aprendizaje o la reflexión personal que otorga el significado después de vivenciar la experiencia (Smith, 2001).

Así pues, este tipo de aprendizaje proporciona un efecto sinérgico, basado en el potencial que tiene la interacción con la propia realidad que favorece un desempeño superior en el propio sujeto al tener que lidiar con problemas que dependen de su propio esfuerzo cognitivo (Raelin, 2000).

La experiencia propuesta en este trabajo, además de su carácter multidisciplinar (psicología, criminología y derecho), se enmarca dentro del conjunto de técnicas de aprendizaje activas, progresivas y consecutivas, desarrollándose el aprendizaje cooperativo, la simulación y el aprendizaje experiencial aplicados a una situación real.

Para llevar a cabo dicho proyecto se seleccionó un tema de actualidad, de gran importancia y donde las políticas criminales sufren continuas modificaciones, dado su carácter cambiante y dinámico, el "Child Grooming”. Este fenómeno aparece hoy en día como parte de nuestra realidad debido a que pertenecemos a un mundo globalizado donde internet y las redes sociales crecen y cambian de manera exponencial y se extienden a un ritmo increíblemente rápido $(\mathrm{Li}, 2006)$.

Cuando es un adulto quien aprovecha los recursos de las nuevas tecnologías para engatusar a menores, y provocar encuentros sexuales fuera del ciberespacio, este comportamiento es considerado como "Child Grooming” (Berson, 2003; Brown, 2001; Gillespie, 2002; Monge Fernández, 2010). Este proceso de embaucamiento o seducción por parte de un adulto se realiza mediante las TRIC (Tecnologías de la Información, Relación y Comunicación), es decir, mediante el uso de las redes sociales, como puede ser Facebook, Instagram, etc que tan habituales son entre los nativos digitales (Prensky, 2001)

Es por tanto una necesidad fomentar el adecuado conocimiento y las estrategias de afrontamiento necesarias entre nuestros jóvenes que les posibiliten hacer frente debidamente a este tipo de situaciones en las que se pueden ver implicados. Y para lograr este propósito es necesario desarrollar programas de intervención adecuados e implantar las acciones necesarias que sean capaces de minimizar, si no eliminar totalmente, estas amenazas en los centros escolares.

Siguiendo estos dos propósitos se planteó una actividad basada en el aprendizaje experiencial que fuera capaz de fomentar este tipo de metodologías de innovación educativa entre nuestros estudiantes universitarios, dándoles la oportunidad de vivenciar lo aprendido y de hacerlos responsables y protagonistas de su propio aprendizaje a la vez que conseguíamos concienciar, sensibilizar y formar a jóvenes ante el fenómeno del Grooming.

Este proyecto llamado INTUYE EL “CHILD GROOMING” se enmarcó dentro de la asignatura Víctímología, de $3^{\circ}$ curso del Doble Grado en Psicología y Criminología, vinculada con Psicología Criminal y Derecho Penal II. Participaron 12 alumnos, divididos 
en 4 grupos de 3 estudiantes cada uno, quienes llevaron a cabo el diseño, la planificación, la elaboración y el desarrollo de una estrategia de prevención primaria en una escuela valenciana con niños de $5^{\circ}$ y $6^{\circ}$ de primaria, dos grupos por curso.

Este trabajo se evaluó en su totalidad, formando parte de los trabajos prácticos de la asignatura: trabajo de investigación, realización de un programa de prevención y exposición oral.

\section{Objetivos}

Los objetivos de este proyecto son:

- Relacionar los contenidos teórico y prácticos de varias asignaturas para el desarrollo posterior de una actividad.

- Planificar, diseñar y elaborar una intervención de prevención primaria en la escuela que permita experimentar lo aprendido en el aula.

- Practicar las habilidades de comunicación e interacción con un determinado público externo.

- Desarrollar la intervención en una situación real que ofrezca el contexto necesario para reflexionar y enfrentarse a una futura situación profesional.

- Reflexionar sobre la utilidad del trabajo realizado.

- Sensibilizar a los menores de los peligros del mal uso de las nuevas tecnologías.

\section{Desarrollo de la innovación}

Dentro de la asignatura "Victimología", es donde se desarrolla la actividad descrita en el presente trabajo. Una vez impartidos los conocimentos sobre la prevención de la victimización en personas vulnerables, en especial los relacionados con menores víctimas del acoso o abuso sexual vía internet, se trabajan los tipos de estrategias o medidas de prevención primaria posibles a desarrollar en el ámbito de la escuela. Todo ello unido a contenidos recibidos en otras asignaturas, como la Psicología Criminal, donde se estudia el perfil del agresor y su funcionamiento para cometer el delito; y Derecho Penal II, donde se trabaja las tipologías delictivas relacionadas con las agresiones y abusos sexuales en los menores.

En un primer momento se trabajó en el aula mediante actividades de aprendizaje cooperativo, donde los grupos de estudiantes se encontraban estrechamente vinculados para elaborar el proyecto propuesto en el ámbito de la prevención primaria en la escuela. Este entrenamiento permite desarrollar competencias académicas y profesionales, además de habilidades interpersonales (Fernández, 2006).

En un segundo momento, el aula se transformó en un escenario práctico, donde los alumnos pasaron a ser protagonistas de su propio aprendizaje. Aquí es donde se desarrolla el aprendizaje práctico mediante la "simulación”. Esta técnica, ofrece al alumnado "un marco

(cc) EY-NC-ND 2018, Universitat Politècnica de València

Congreso IN-RED (2018) 
donde aprender de manera interactiva por medio de una experiencia viva, afrontar situaciones que quizá no están preparados para superar en una situación real, expresar sus sentimientos respecto al aprendizaje y experimentar con nuevas ideas y procedimientos" (Fernández, 2006, p.50). El profesorado ofreció pautas desde la primera fase de la simulación hasta la última, dada la importancia de que el progreso de las tareas se desarrollara eficazmente, puesto que el éxito de la posterior práctica en la situación real, depende, en gran parte, del trabajo realizado en este tipo de aprendizajes (Crookall y Oxford, 1990).

Para finalizar, se desarrolló el propio aprendizaje experiencial “real”, es decir, se trasladó el aula a una situación no simulada. De esta manera se involucra al individuo en una interacción directa con aquello que se está estudiando, y a un lugar donde puede practicarlo con agentes reales. Ya se deja en el aula la contemplación o descripción intelectual.

Una vez acabado el proyecto, este finaliza en el aula, con una sesión para asegurar el aprendizaje, de manera que el alumno se sienta íntimamente ligado a un proceso de reflexión personal, construyendo significados a partir de la experiencia vivida (Smith, 2001, citado en Ariza, 2010). Además, como culminación del trabajo, los participantes entregaron un informe escrito con la investigación y descripción del trabajo realizado, por lo que se evaluó en su totalidad, formando parte de los trabajos prácticos de la asignatura: trabajo de investigación (20\%), realización de un programa de prevención (30\%)y exposición oral (10\%), lo que hacía un total del 50\% de la asignatura.

A continuación, se explicitan las fases del proyecto:

\subsection{Fases de la intervención:}

El programa para realizar la intervención INTUYE EL “CHILD GROOMING”, se dividió en 4 fases y 7 sesiones, dos en cada fase, excepctuando la $1^{\text {a }}$ que solo tenía una sesión.

Fase 1: En primer lugar, se estableció una reunión con la dirección del centro escolar para analizar la necesidad de la intervención y establecer los contenidos relacionados con el fenómeno del Child Grooming. A este acto acudieron tres representantes del alumnado, para que su aprendizaje comenzara desde la negociación y posibilidades de realizar la intervención.

Fase $2^{\circ}$ Aprendizaje cooperativo en aula, se marcaron los objetivos a conseguir, se ofrecieron directrices básicas para la elaboración del proyecto, se organizaron los grupos y distribuyeron las responsabilidades y tareas que cada alumno tenía que desarrollar en lo referido a elaboración de contenidos y material con el que se iba a desarrollar la intervención, adaptándola al alumnado al que iba dirirgida, según edad y conocimientos previos, etc. 
$\underline{\text { Fase } 3^{\mathbf{0}}}$. Simulación. Antes de iniciar la simulación propiamente dicha, se comprobó que todos los participantes tuvieran claro en qué iba a consistir y con qué instrumentos contaban para desarrollar la situación planteada, de forma que pudieran debatir sobre ella y adoptar las soluciones que cada participante del grupo estimara oportunas. A continuación, comenzó la simulación y otro grupo de estudiantes se convirtieron en observadores. También se entrenaron y pusieron en práctica las estrategias más adecuadas de comunicación. Al finalizar la segunda simulación, se abrió un debate para tratar cuestiones que se podían mejorar, modificar, ampliar, etc., siempre con el docente como guía. De esta manera, se permitió a los aprendices proporcionar su propio feedback, comentando lo sucedido y planteando sus dudas, inseguridades, etc.

Fase $4^{\mathbf{0}}$. Aprendizaje experiencial real. Desarrollo de la intervención durante dos sesiones de 45 minutos llevada a cabo por tres estudiantes en cada uno de los grupos, conducida en todo momento por el docente de la asignatura. En la Figura 1, se puede observar a un grupo de universitarios en uno de los momentos de dicha intervención.

Sesión final. Esta sesión se desarrolló en el aula como reflexión del trabajo realizado, dudas y otras cuestiones para su mejora y entrega de un informe escrito con la descripción de la herramienta de prevención para su posterior evaluación como parte práctica de la asignatura.

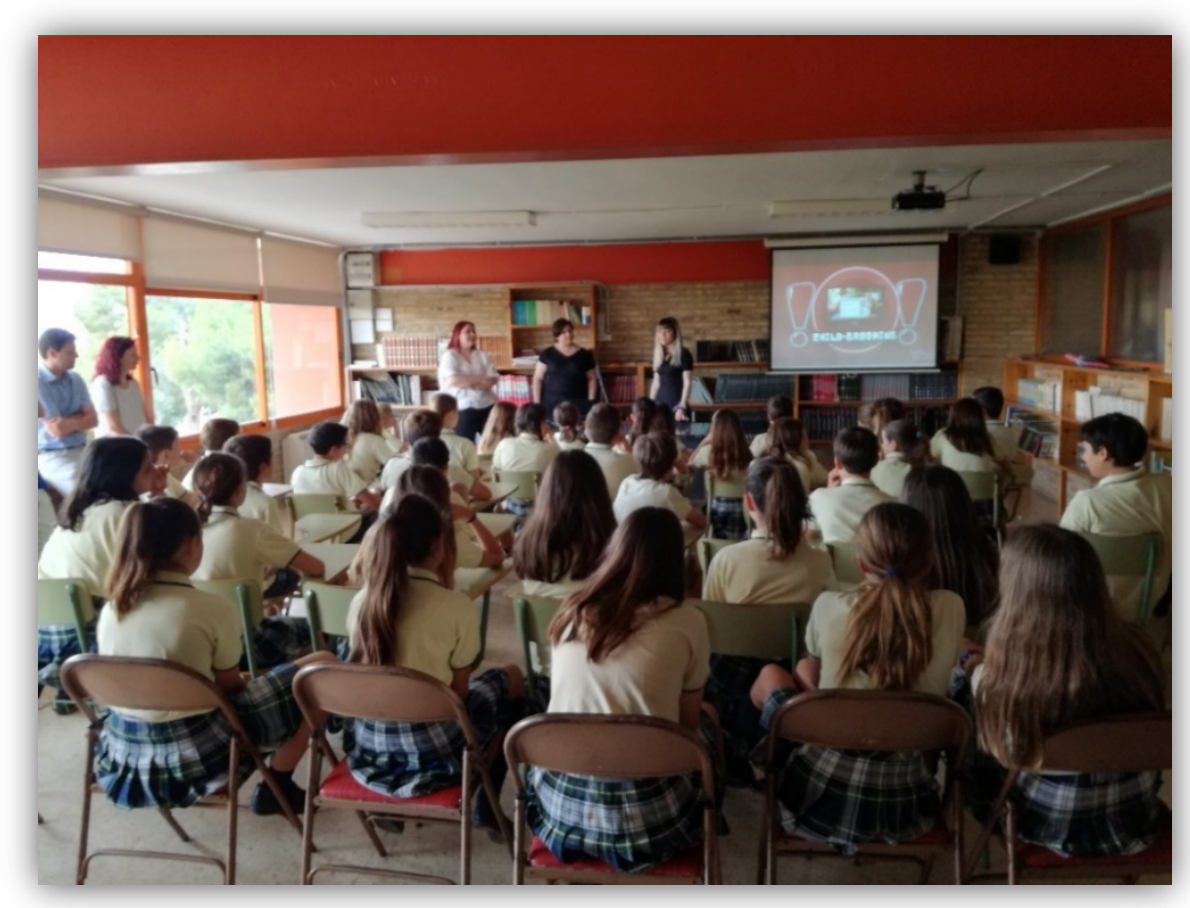

Figura 1. Situación real de la intervención “Child Grooming” en el Colegio Alfinach. 


\subsection{Cronograma de la metodología}

En la Figura 2 se puede observar, de forma más visual, la distribución de las sesiones del INTUYE EL “CHILD GROOMING”, desarrolladas en semanas consecutivas.
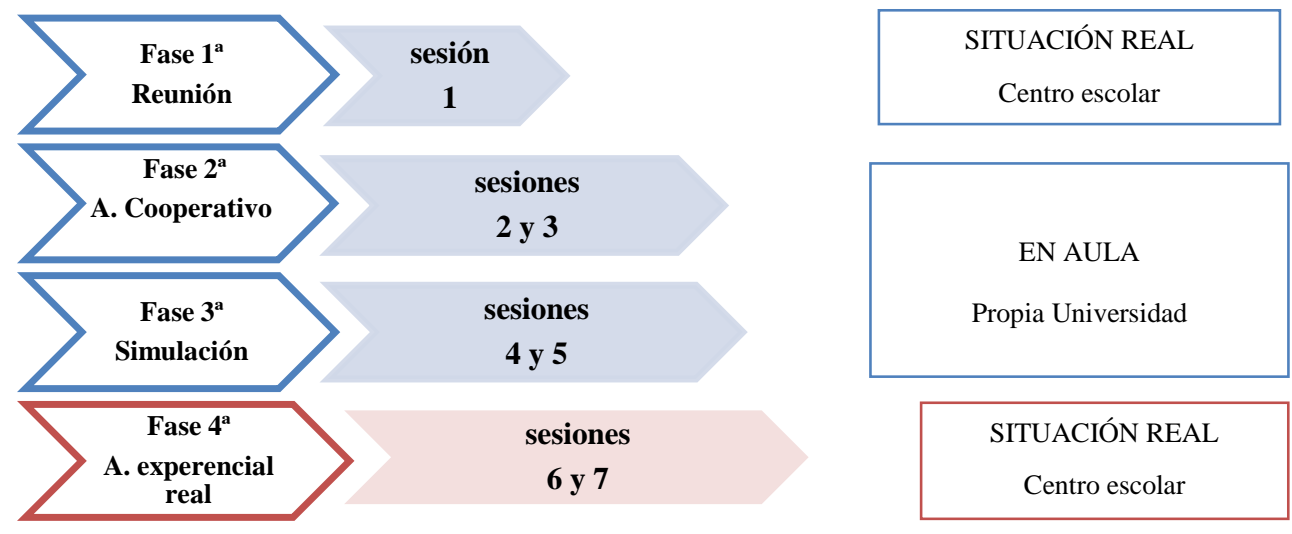

Figura 2. Cronograma de las sesiones que componen la intervención.

\subsection{Material utilizado}

A continuación se presentan algunas de las diapositivas del material empleado, por uno de los grupos de universitarios, para el desarrollo de INTUYE EL "CHILD GROOMING" (Figura 3):

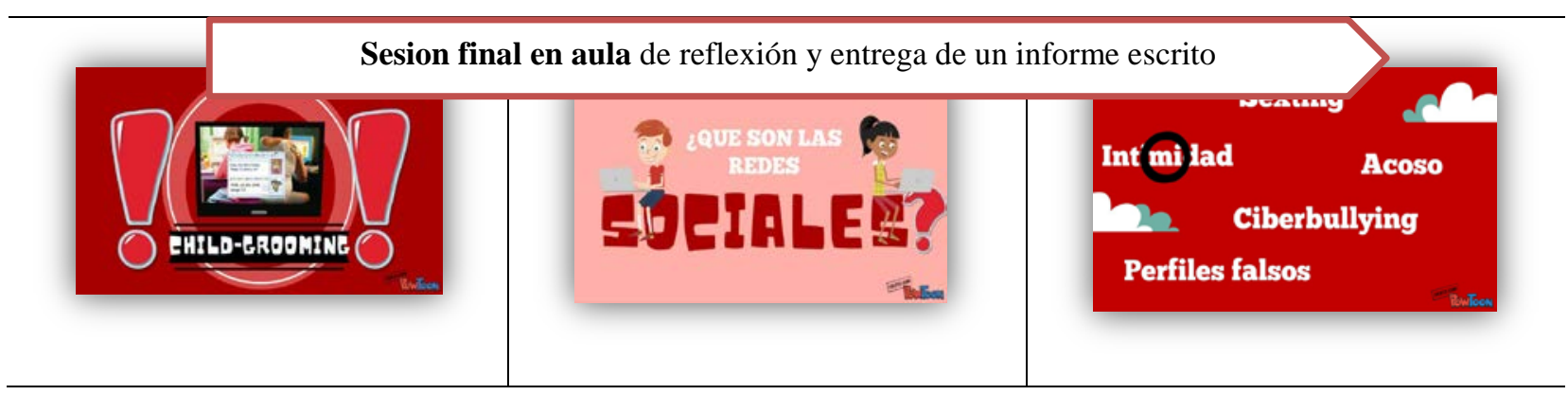

(c)) EY-NG-ND 2018, Universitat Politècnica de València

Congreso In-Red (2018) 


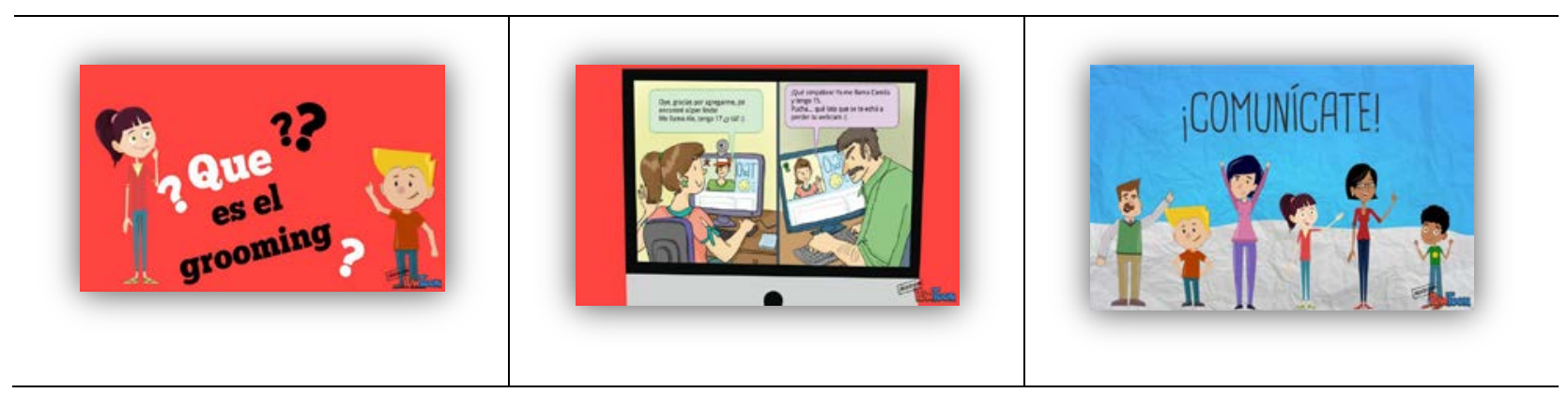

Figura 3. Ejemplo de 6 diaptositivas de la intervención INTUYE EL “CHILD GROOMING”

\section{Resultados}

Los resultados de evaluación de la intervención por parte de los 12 estudiantes universitarios fueron muy satisfactorios, según manifestaron en el cuestionario compuesto por 10 ítems, con tipo de respuesta cuantitativa, con valoraciones entre 0 a 10 puntos. Dicho cuestionario mide variables relacionadas con el aprendizaje de las asignaturas, en conocimiento teóricos y prácticos, así como para la futura profesión del estudiante; el aprendizaje en habilidades comunicativas; la percepción de utilidad de la intervención para la comunidad; la motivación que ha provocado en el estudiante desarrollar el proyecto; la recomendación de la actividad; así como la satisfacción personal. En la Figura 4, se muestran los resultados obtenidos sobre las medias de las puntuaciones ofrecidas por los

\section{Resultados evaluación actividad experiencial}

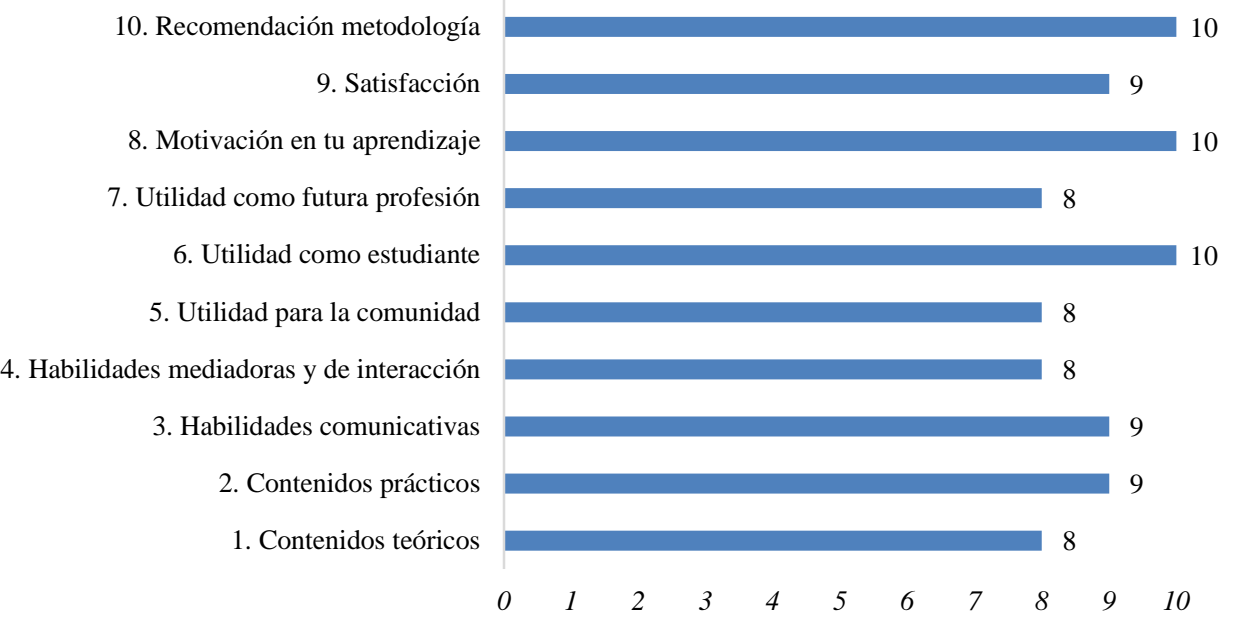

estudiantes.

Figura 4. Resultados de la evaluación presentado los ítems resumidos con las puntuaciones medias de las valoraciones aportadas por los estudiantes. Elaboración propia.

(cc) EY-NC-ND 2018, Universitat Politècnica de València

Congreso IN-RED (2018) 


\section{Conclusiones:}

Este proyecto fue muy motivador para los estudiantes ya que por unanimidad manifestaron que su aprendizaje había sido diferente, más creativo, innovador y vivencial. La propia elaboración de un proyecto vivo en el que ellos podrían actuar y no quedara en una carpeta, ha significado conseguir muchas de las competencias exigidas en el grado, como su autonomía, su capacidad de análisis, de planificación, además del trabajo en equipo. Otras capacidades que se plamaron al finalizar la actividad y como resumen de seguimiento, fue la capacidad de análisis y crítica, reflexión personal, capacidad de extrapolar los conocimientos a la práctica y la capacidad de influencia en la comunidad.

Esta influencia fue comprobada en el momento en que los menores intercambiaban sus pensamientos con los estudiantes universitarios partícipes, consiguiendo sensibilizarles sobre los peligros del mal uso de las nuevas tecnologías. Además, a este hecho, se suma la posterior comunicación con el centro, donde se pudo comprobar la satisfacción de los menores sobre la charla recibida.

Cabe señalar que las notas de los trabajos prácticos de la asignatura fueron mejores y más completas en comparación con años anteriores.

Es fundamental poder fomentar este tipo de prácticas entre los alumnos de grado que les capaciten para poder vivenciar y experimentar situaciones reales que aumenten su seguridad y motivación y favorezcan su interés hacia uno de los campos profesionales en los que la Criminología y la Psicología actúan.

\section{Referencias}

Aparicio, L. y De Paredes, C. (2016). El role-playing o experiencias de simulación como instrumento educativo en los estudios universitarios de Ciencias Sociales. Tamara Ramiro-Sánchez, $\mathrm{M}^{\mathrm{a}}$ Teresa Ramiro-Sánchez, y M Ma Paz Bermúdez (Comp.). XIII Foro Internacional sobre la Evaluación de la Calidad de la Investigación y de la Educación Superior (FECIES). Granada. España.

Ariza, M. R. (2010). El aprendizaje experiencial y las nuevas demandas formativas. Revista de antropología experimental, (10), 89-102.

Benito, Á. y Cruz A. (2005). Nuevas claves para la docencia universitaria: en el espacio europeo de educación superior. Madrid: Narcea Ediciones.

Berson, I. R. (2003). Grooming cybervictims: The psychosocial effects of online exploitation for youth. Journal of School Violence, 2(1), 5-18.

Brown, D. (2001). Developing strategies for collecting and presenting grooming evidence in a high tech world. National Center for Prosecution of Child Abuse. Update, 14(11). 
Chisholm, C.U., Harris, M.S.G., Northwood, D.O. y Johrendt, J.L. (2009). “The Characterisation of Work-Based Learning by Consideration of the Theories of Experiential Learning” en European Journal of Education, III, 44: 319-337.

Crookall, D. y Oxford, R. (1990). Simulation, Gaming and Language Learning. New York: Newbury House Publishers.

De Miguel, M. (2005). Modalidades de Enseñanza centradas en el desarrollo de Competencias: Orientaciones para promover el cambio metodológico en el Espacio Europeo de Educación Superior. Proyecto EA2005-0118. Universidad de Oviedo.

Fernández, A. (2006). Metodologías activas para la formación de competencias. Educatio siglo XXI, 24: 35-5

Gillespie, A. (2002). Child protection on the internet challenges for criminal law. Child and Family Law Quarterly, 14(4), 411-425.

Itin, C.M. (1999). "Reasserting the philosophy of experiential education as a vehicle for change in the 21st Century”. Journal of Experiential Education, 85-97.

Labrador-Piquer, M. J. y Andreu-Andrés, M. A. (2008). Metodologías activas. Valencia: Editorial UPV.

Li, Q. (2006). Cyberbullying in schools a research of gender differences. School Psychology International, 27(2), 157-170.

March, A. F. (2006). Metodologías activas para la formación de competencias. Educatio siglo $X X I, 24,35-56$.

Miguel-Dávila, J., López-Berzosa, D. y Martín-Sánchez, M. (2012). ¿Una participación activa del alumno pronostica una buena nota en el examen? Working Papers on Operations Management, 3(2), 71-83.

Monge, A. (2010). De los abusos y agresiones sexuales a menores de trece años tras la reforma penal de 2010. Revista De Derecho, 15, 85-106.

Prensky, M. (2001). Digital Game-Based Learning. New York: McGraw-Hill.

Raelin, J.A. (2000). Work-based Learning: The New Frontier of Management Development. Nueva Jersey: Prentice Hall Inc.

Smith, M.K. (2001, 2010)). David A Kolb on Experiential Learning, The Encyclopedia of Informal Education. [http://www.infed.org/b-explrn.htm. Recuperado: 02-122017.

Zabalza, M.A. (2011), Metodología docente, REDU: Revista de Docencia Universitaria, Vol. 9, Nº 3, 2011 (Ejemplar dedicado a: El espacio europeo de educación superior. ¿Hacia dónde va la Universidad Europea?) p.81. 